\title{
A simple model for mantle-driven flow at the top of Earth's core
}

\author{
Hagay Amit ${ }^{1}$, Julien Aubert ${ }^{2}$, Gauthier Hulot $^{1}$, and Peter Olson ${ }^{3}$ \\ ${ }^{1}$ Equipe de Géomagnétisme, Institut de Physique du Globe de Paris (Institut de Recherche associé au CNRS et à l' Université Paris 7), \\ 4 Place Jussieu, 75252 Paris Cedex 05, France \\ ${ }^{2}$ Equipe de Dynamique des Systèmes Géologiques, Institut de Physique du Globe de Paris \\ ${ }^{3}$ Department of Earth and Planetary Sciences, Johns Hopkins University, Baltimore, MD 21218, USA
}

(Received November 15, 2007; Revised April 7, 2008; Accepted April 26, 2008; Online published September 8, 2008)

\begin{abstract}
We derive a model for the steady fluid flow at the top of Earth's core driven by thermal coupling with the heterogeneous lower mantle. The model uses a thermal wind balance for the core flow, and assumes a proportionality between the horizontal density gradients at the top of the core and horizontal gradients in seismic shear velocity in the lowermost mantle. It also assumes a proportionality between the core fluid velocity and its radial shear. This last assumption is validated by comparison with numerical models of mantle-driven core flow, including self-sustaining dynamo (supercritical) models and non-magnetic convection (subcritical) models. The numerical dynamo models show that thermal winds with correlated velocity and radial shear dominate the boundary-driven large-scale flow at the top of the core. We then compare the thermal wind flow predicted by mantle heterogeneity with the 150 year time-average flow obtained from inverting the historical geomagnetic secular variation, focusing on the non-zonal components of the flows because of their sensitivity to the boundary heterogeneity. Comparing magnitudes provides an estimate of the ratio of lower mantle seismic anomalies to core density anomalies. Comparing patterns shows that the thermal wind model and the time-average geomagnetic flow have comparable length scales and exhibit some important similarities, including an anticlockwise vortex below the southern Indian and Atlantic Oceans, and another anticlockwise vortex below Asia, suggesting these parts of the non-zonal core flow could be thermally controlled by the mantle. In other regions, however, the two flows do not match well, and some possible reasons for the dissimilarity between the predicted and observed core flow are identified. We propose that better agreement could be obtained using core flows derived from geomagnetic secular variation over longer time periods.
\end{abstract}

Key words: Thermal wind, mantle tomography, time-average core flow, geodynamo.

\section{Introduction}

Convection in the outer core is likely influenced by the heterogeneous thermal boundary conditions imposed on the core by the overlying mantle. Evidence for this mantle influence is sometimes found in paleomagnetic and archeomagnetic field models in the form of long-term departures from axial symmetry (Gubbins and Kelly, 1993; Johnson and Constable, 1995; Constable et al., 2000; Korte et al., 2005), but not always (McElhinny et al., 1996; Carlut and Courtillot, 1998; Hongre et al., 1998) because such departures are difficult to identify unambiguously (Bouligand $e t$ al., 2005; Hulot and Bouligand, 2005; Khokhlov et al., 2006). In this paper we focus on historical time-scales to assess the influence of the overlying lower mantle on the current working of the geodynamo. More specifically, we study core flows rather than the geomagnetic field itself because the time-scale of flow variations is shorter than the time-scale of the geomagnetic field (Hulot and LeMouël, 1994; Le Huy et al., 2000), potentially giving better chances of identifying steady features by averaging core flows over historical times than by averaging the geomagnetic field.

Copyright (c) The Society of Geomagnetism and Earth, Planetary and Space Sciences (SGEPSS); The Seismological Society of Japan; The Volcanological Society of Japan; The Geodetic Society of Japan; The Japanese Society for Planetary Sciences; TERRAPUB.
We therefore explore the possibility that flows driven by the heterogeneous lower mantle could explain some of the central features found in time-average core surface flow models accounting for the historical geomagnetic secular variation. The latter flow models testify for significant asymmetries within the core. First, most of the core flow activity, as well as the secular variation, occurs in the Atlantic hemisphere, while the Pacific hemisphere remains much quieter (Amit and Olson, 2006). Second, core flow models averaged over 150 years display significant north-south asymmetry, with strong westward drift at low and mid-latitudes of the southern hemisphere, but much less drift in the northern hemisphere (Pais and Hulot, 2000; Amit and Olson, 2006). Do these asymmetries merely reflect transient short time-scale features of the geodynamo, or could they indeed be caused by the heterogeneity of the overlying mantle?

It has already been proposed that heterogeneous mantle boundary conditions could control some of the fluid motion in the outer core (Bloxham and Gubbins, 1987). Most recent studies relied on numerical simulations and assumed thermal boundary conditions related to seismic shear velocity anomalies in the lower mantle (see Aubert et al., 2007, and references therein). According to this assumption, a high/low seismic shear velocity anomaly at the lower mantle correlates with a dense/light (cold/hot) lower mantle 
(Yuen et al., 1993; Forte and Mitrovica, 2001), and therefore a high/low heat flux across the core-mantle boundary. This for instance led Olson and Christensen (2002) to find that long-term time-average flows in full 3D self-consistent numerical dynamos with such tomographic boundary conditions are indeed affected by both mantle-driven and internal core dynamical effects, and as a result, display more westward drift in the southern than in the northern hemisphere, as well as a large anti-clockwise vortex below the southern Atlantic and Indian Oceans. These are akin to similar features seen in short-term time-average core flow models derived from geomagnetic secular variation models (Pais and Hulot, 2000; Amit and Olson, 2006). More recently, Aubert et al. (2007) used numerical dynamos to study the likelihood of identifying mantle control on core flows averaged over such short time periods, namely three vortex turnover times which they claim is equivalent to 100-360 years of observations. Their results show that a significant signature of mantle-driven thermal wind can be expected in core flows averaged over such short periods. Aubert et al. (2007), however, did not directly assess the extent to which the time-average core flow computed from the historical geomagnetic data displays the kind of features expected from mantle controlled core flow. The purpose of this study is precisely to look into this in details.

To infer the expected signature of mantle control on core surface flows we use a simple model starting from thermal wind theory, which relies on a vorticity balance at the top of the core dominated by effects of rotation (Section 2). In addition, we assume that thermal boundary conditions imposed by the mantle induce lateral density gradients at the top of the core proportional to the seismic shear velocity pattern in the lower mantle. Those density gradients drive a thermal wind flow which we further assume has a radial shear proportional to the flow itself. This approach has already been used by Amit and Olson (2006) to examine mantle control on their zonal time-average core flow inferred from the geomagnetic secular variation. They found that the north-south asymmetry in mid-latitudes of the zonal geomagnetic flow is qualitatively similar to that of the zonal thermal wind, and may therefore be an indication to mantle control. However, the difficulty in separating the effects of the homogeneous dynamo flow and the mantle-driven flow might interfere with such an interpretation. Here we focus on the non-zonal component of the flow, which best reflects the signature of mantle control (by avoiding background zonal dynamo flows unrelated to mantle control). This mantle-driven thermal wind flow model is first validated with the help of flow models derived from numerical dynamos (Section 3). It is next used for comparisons with the time-average core flow model of Amit and Olson (2006) based on geomagnetic secular variation inversions (Section 4). This finally allows us to discuss the possibility that thermal control of the mantle could be responsible for some of the features found in the time-average core flow model inferred from 150 years of geomagnetic data, as Aubert et al. (2007) suggested could be the case.

\section{Mantle-driven Thermal Wind Flow Model}

In a spherical coordinate system $(r, \theta, \phi)$, where $r$ is the distance from Earth's center, $\theta$ is co-latitude and $\phi$ is longitude, the standard thermal wind equation for a thick fluid shell just below the core-mantle boundary $(r=R)$ is (Pedlosky, 1987)

$$
\frac{\partial \mathbf{u}}{\partial z}=\frac{g_{0}}{2 \Omega \rho_{0}}(\nabla \rho \times \hat{\mathbf{r}})
$$

where $\mathbf{u}$ is velocity, $z$ is the direction of the rotation axis, $g_{0}$ and $\rho_{0}$ are gravitational acceleration and density at the core-mantle boundary respectively, $\Omega$ is rotation rate, $\rho$ is the laterally-variable density and $\hat{\mathbf{r}}$ is the radial unit vector. In spherical coordinates (1) can be rewritten as

$$
\cos \theta \frac{\partial \mathbf{u}}{\partial r}-\frac{\sin \theta}{R} \frac{\partial \mathbf{u}}{\partial \theta}=\frac{g_{0}}{2 \Omega \rho_{0}}(\nabla \rho \times \hat{\mathbf{r}})
$$

which implies:

$$
\cos \theta \frac{\partial u_{\phi}}{\partial r}-\frac{\sin \theta}{R} \frac{\partial u_{\phi}}{\partial \theta}=-\frac{g_{0}}{2 \Omega \rho_{0}} \frac{1}{R} \frac{\partial \rho}{\partial \theta}
$$

and (because $u_{r}=0$ at the core surface):

$$
\cos \theta \frac{\partial u_{\theta}}{\partial r}-\frac{\sin \theta}{R} \frac{\partial u_{\theta}}{\partial \theta}=\frac{g_{0}}{2 \Omega \rho_{0}} \frac{1}{R \sin \theta} \frac{\partial \rho}{\partial \phi}
$$

Our goal is to solve for the velocity components on the left hand sides of (3)-(4) given some knowledge of $\rho$ (which we will later infer independently). The second term on the left hand sides of (3)-(4) (the meridional gradient of the flow, $\partial u_{\phi} / \partial \theta$ and $\partial u_{\theta} / \partial \theta$ ) can be integrated for a given density pattern provided the first term on the left hand sides of (3)-(4) (the radial shear $\partial u_{\phi} / \partial r$ and $\partial u_{\theta} / \partial r$ ) is known. Unfortunately this is not directly the case. To our best knowledge, there is neither a general analytical solution to (3)-(4), nor a general theoretical relation between the radial shear and the flow. However, and as we will later show, numerical dynamos suggest a very convenient approximate proportionality between the mantle-driven flow and its radial shear at the top of the core:

$$
\frac{\partial \mathbf{u}_{h}}{\partial r} \simeq L^{-1} \frac{\mathbf{u}_{h}}{R}
$$

where $L^{-1}$ is a non-dimensional parameter. Substituting (5) into (3)-(4), the thermal wind at the top of the core is then approximated by

$$
\begin{gathered}
L^{-1} \cos \theta u_{\phi}-\sin \theta \frac{\partial u_{\phi}}{\partial \theta}=-\frac{g_{0}}{2 \Omega \rho_{0}} \frac{\partial \rho}{\partial \theta} \\
L^{-1} \cos \theta u_{\theta}-\sin \theta \frac{\partial u_{\theta}}{\partial \theta}=\frac{g_{0}}{2 \Omega \rho_{0}} \frac{1}{\sin \theta} \frac{\partial \rho}{\partial \phi}
\end{gathered}
$$

To infer the density $\rho$ driving the thermal wind as a result of thermal core-mantle coupling, we begin with the standard assumption that the heterogeneous heat flux across the core-mantle boundary is proportional to seismic shear velocity anomalies at the lowermost mantle (Olson and Glatzmaier, 1996; Glatzmaier et al., 1999; Olson and Christensen, 2002; Christensen and Olson, 2003). We further 
Table 1. Global vector correlation coefficient $C_{s}$ between non-zonal radial shear ${\frac{\partial \mathbf{u}_{h}}{\partial r}}^{\mathrm{nz}}$ and non-zonal normalized flow ${\frac{\mathbf{u}_{h}}{R}}^{\mathrm{nz}}$ at the top of the free stream $\left(r=0.938\right.$ for $E=3 \cdot 10^{-4}$ and $r=0.905$ for $\left.E=6 \cdot 10^{-4}\right)$ and their ratio $L^{-1}$. Also given the high-latitudes correlation coefficients $C_{45}$ for latitudes higher than $45^{\circ}$ and $C_{60}$ for latitudes higher than $60^{\circ}$. The global correlation coefficient $C_{\mathrm{c}}$ is between the non-zonal radial shear term $\cos \theta{\frac{\partial \mathbf{u}_{h}}{\partial r}}^{\mathrm{nz}}$ and the non-zonal normalized flow term $\cos \theta{\frac{\mathbf{u}_{h}}{R}}^{\mathrm{nz}}$ and their ratio is $L_{\mathrm{c}}^{-1}$. Finally, a global correlation coefficient $C_{\rho}$ between the non-zonal imposed boundary heat flux and the non-zonal density at the top of the free stream is also given. The correlation coefficients are calculated as the averaged scalar product of the two vectors normalized by their averaged magnitudes. The ratios $L^{-1}$ and $L_{\mathrm{c}}^{-1}$ are calculated as the best-fit global linear regression. The parameters used in the dynamo simulations are the Rayleigh $(R a)$, Ekman $(E)$, Prandtl (Pr) and magnetic Prandtl (Pm) numbers. The magnitude of the heat flux anomaly imposed on the outer boundary is $q^{*}$ defined as the ratio of peak-to-peak to mean heat flux. All cases have tomographic boundary conditions, except cases $Y_{2}^{2}$ and $Y_{2}^{1}$ that have corresponding single-harmonic boundary conditions. All cases have been time-averaged over several magnetic diffusion times. All cases are supercritical dynamos except $S$ which is subcritical. All dynamos are dipolar except $T 2$.

\begin{tabular}{cccccccccccccc}
\hline Case & $q^{*}$ & $R a$ & $E$ & $P r$ & $P m$ & $C_{s}$ & $L^{-1}$ & $C_{45}$ & $C_{60}$ & $C_{\mathrm{c}}$ & $L_{\mathrm{c}}^{-1}$ & $C_{\rho}$ \\
\hline$T 1 l q$ & 0.1 & $6 \cdot 10^{5}$ & $6 \cdot 10^{-4}$ & 1 & 4 & 0.41 & 0.96 & 0.42 & 0.69 & 0.48 & 2.36 & 0.68 \\
$T 2 l q$ & 0.1 & $1 \cdot 10^{6}$ & $6 \cdot 10^{-4}$ & 1 & 4 & 0.11 & 1.80 & 0.44 & 0.75 & 0.43 & 2.35 & 0.51 \\
$T 0$ & 0.5 & $1 \cdot 10^{6}$ & $3 \cdot 10^{-4}$ & 1 & 3 & 0.61 & 5.05 & 0.66 & 0.71 & 0.65 & 3.80 & 0.77 \\
$T 1$ & 0.5 & $2.5 \cdot 10^{6}$ & $3 \cdot 10^{-4}$ & 1 & 2 & 0.54 & 3.95 & 0.61 & 0.66 & 0.64 & 3.65 & 0.64 \\
$T 1 h p$ & 0.5 & $2.5 \cdot 10^{6}$ & $3 \cdot 10^{-4}$ & 1 & 5 & 0.53 & 3.22 & 0.63 & 0.76 & 0.64 & 3.23 & 0.73 \\
$T 2$ & 0.5 & $1 \cdot 10^{7}$ & $3 \cdot 10^{-4}$ & 1 & 2 & -0.05 & 3.83 & 0.46 & 0.71 & 0.34 & 2.98 & 0.76 \\
$S$ & 0.5 & $2.5 \cdot 10^{3}$ & $3 \cdot 10^{-4}$ & 1 & 2 & 0.16 & 3.81 & 0.64 & 0.75 & 0.59 & 3.50 & 0.90 \\
$Y_{2}^{2}$ & 0.625 & $2.5 \cdot 10^{6}$ & $3 \cdot 10^{-4}$ & 1 & 2 & 0.24 & 1.37 & 0.47 & 0.67 & 0.48 & 2.20 & 0.71 \\
$Y_{2}^{1}$ & 0.625 & $2.5 \cdot 10^{6}$ & $3 \cdot 10^{-4}$ & 1 & 2 & 0.49 & 2.06 & 0.55 & 0.70 & 0.57 & 2.33 & 0.96 \\
\hline
\end{tabular}

assume that the density anomaly at the top of the core is itself proportional to this heterogeneous heat flux (Gubbins, 2003; Amit and Olson, 2006). These imply the following linear relationship between horizontal density gradients at the top of the core and horizontal shear wave velocity gradients at the base of the mantle:

$$
\left.\frac{1}{\rho_{0}} \nabla_{h} \rho\right|_{\text {core }}=\left.\frac{C}{v_{s}} \nabla_{h} v_{s}\right|_{\text {mantle }}
$$

where $v_{s}$ is seismic shear wave velocity in the mantle, $\rho_{0}$ is the mean outer core density, and $C$ is a constant of proportionality. Because the constant $C$ is a priori unknown, in this study mainly flow patterns will be investigated. An estimate of $C$ will nevertheless be derived.

In practice, in order to estimate the thermal wind core surface flow associated with a given pattern of seismic shear velocity anomalies at the lowermost mantle, we use (8) to infer $\rho$, and (6)-(7) to infer the tangential velocity components. We use a central finite-difference numerical method on a regular $5^{\circ} \times 5^{\circ}$ grid. The solution is obtained by a standard matrix inversion scheme. For more details including boundary conditions see Appendix A. We finally focus on the non-zonal component of this model, and we term it from hereafter the thermal wind model.

\section{Model Validation}

The thermal wind model heavily relies on two important assumptions, formulated as (5) and (8). To assess the validity of those assumptions, several tests can be performed using numerical dynamos subjected to heterogeneous boundary conditions (for a detailed description of the numerical dynamo model we use, see Aubert et al., 2007). The pattern of imposed heat flux on the outer boundary is either based on the lower mantle tomography model of Masters et al. (2000) (truncated at degree 8 to consider only robust features) or implemented as a single-harmonic boundary condition. In all cases, we examine flows at the top of the free stream below the Ekman boundary layer, at the depth at which viscous forces become negligible in the force balance. For the simulations discussed here this corresponds to about 3.5-4h $h_{\mathrm{ek}}$ (where $h_{\mathrm{ek}}=\sqrt{E} D$ is the Ekman boundary layer thickness, $E$ is the Ekman number and $D$ is the shell thickness). Those flows are next long-term time averaged to isolate the steady flow. As already stated, we also restrict ourselves to the non-zonal part of the flow to avoid the purely zonal long-term time-average dynamo homogeneous flow (Aubert, 2005) and to focus on mantle-driven core flow features.

For each simulation we assess the validity of (5) by comparing the resulting $\mathbf{u}_{h}^{\mathrm{nz}} / R$ flow with its radial shear $\partial \mathbf{u}_{h}^{\mathrm{nz}} / \partial r$ and computing correlation coefficients and best-fit linear regression values (Table 1). The global correlations between the non-zonal radial shear of the flow $\partial \mathbf{u}_{h}^{\mathrm{nz}} / \partial r$ to the non-zonal tangential flow itself $\mathbf{u}_{h}^{\mathrm{nz}} / R$ at the top of the free stream for cases $T 1 l q, T 0, T 1, T 1 h p$ and $Y_{2}^{1}$ are well above the $95 \%$ significance level, which is about 0.2 for the resolution of the numerical dynamos in this study (Rau et al., 2000). When only high-latitudes are considered, the correlations are high for all cases. For the highest $45^{\circ}$ latitudes all cases yield $0.66>C_{45}>0.42$, and the range is $0.76>C_{60}>0.66$ for the highest $60^{\circ}$ latitudes. It is important to stress that the inferior correlation at low-latitudes is not a major issue for thermal wind modelling because the radial shear term is less important at low-latitudes. Indeed, comparing maps of the relevant quantities for thermal wind modelling, the non-zonal radial shear term $\cos \theta \partial \mathbf{u}_{h}^{\mathrm{nz}} / \partial r$ and the non-zonal tangential flow term $\cos \theta \mathbf{u}_{h}^{\mathrm{nz}} / R$ (Fig. 1), reveals good global pattern agreement for both tangential components. The global correlations between these two terms are high for all cases, $0.64>C_{\mathrm{c}}>0.34$ (Table 1). Note that this approximate relation between the non-zonal time-average radial shear and the non-zonal time-average tangential flow is not a boundary layer effect but rather appears at the top of the free stream. In fact, it can be shown that inside an Ekman boundary layer the correlation decreases until a value of -1 on approach to the outer bound- 

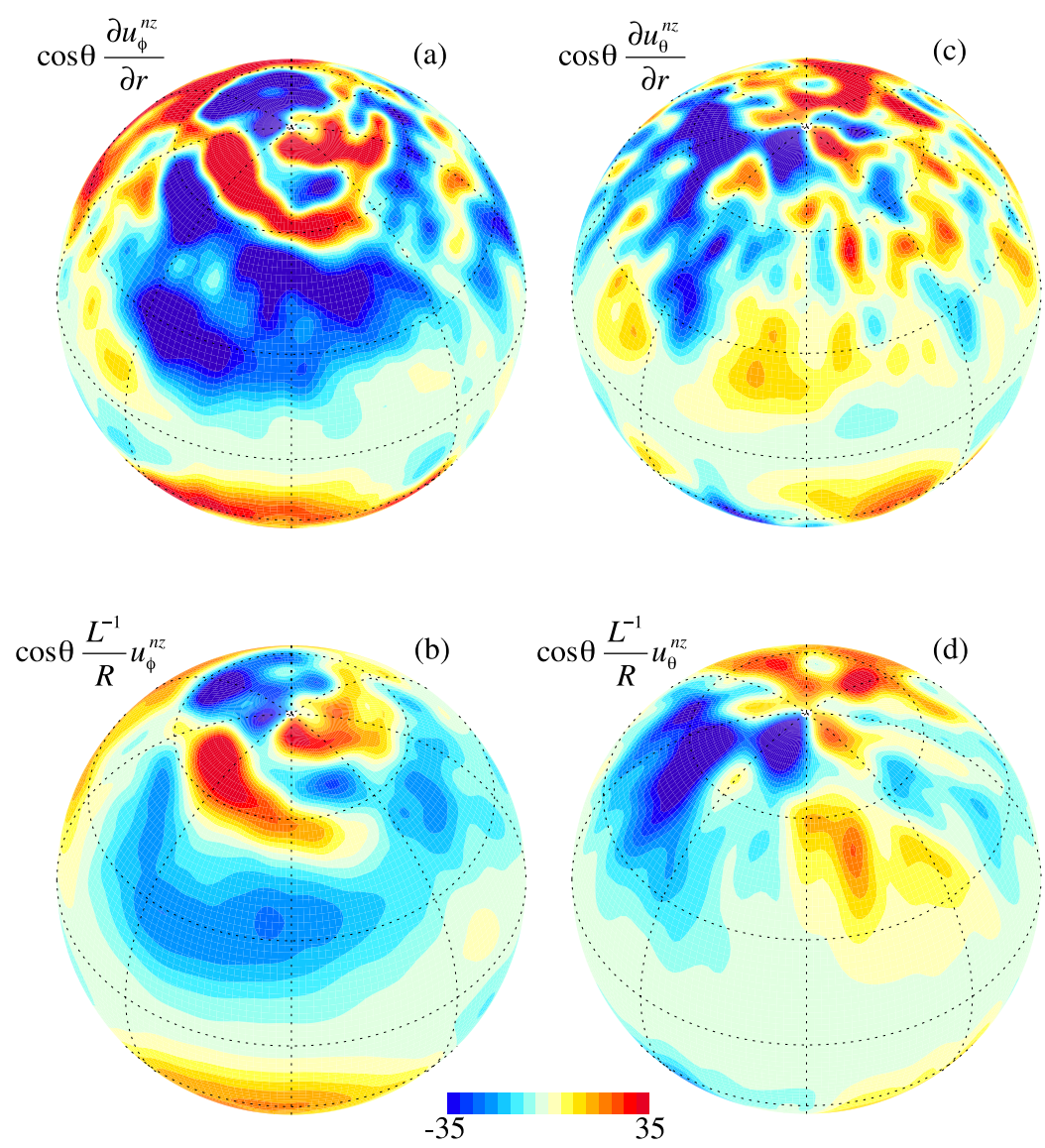

Fig. 1. (a) Non-zonal time-average radial shear of azimuthal flow term $\cos \theta \frac{\partial u_{\phi}}{\partial r}{ }^{\mathrm{nz}}$; (b) Normalized non-zonal time-average azimuthal flow term $\cos \theta \frac{L^{-1}}{R} u_{\phi}{ }^{\mathrm{nz}}$; (c) Non-zonal time-average radial shear of meridional flow term $\cos \theta \frac{\partial u_{\theta}}{\partial r}{ }^{\mathrm{nz}}$; (d) Normalized non-zonal time-average meridional flow term $\cos \theta \frac{L^{-1}}{R} u_{\theta}{ }^{\mathrm{nz}}$. All maps are for case $T 1$ (see Table 1) at the top of the free stream $(r=0.938)$. Red/blue denotes eastward/westward radial shears (a) and flows (b), or southward/northward radial shears (c) and flows (d). All quantities are in dimensionless units.

ary. The high positive correlations reported here are typically found between depths of $\sim 0.05-0.2 D$ (or $\sim 3-12 h_{\mathrm{ek}}$ ), including just below the Ekman boundary layer, the relevant depth for comparison with the time-average core flow computed from the geomagnetic data.

The approximate relation (5) holds for the time-average non-zonal part of the flow because of permanent radial plumes that form at the top of the core as a result of convection driven from above. This produces tangential velocities which reach their radial peak value just below the outer boundary layer. Indeed, radial profiles of nonzonal radial and non-zonal tangential time-average flows (not shown here) generally contain intense radial flows in mid-shell which convert to increasing tangential flows when approaching the outer boundary.

Some cases satisfy (5) globally whereas others tend to fail at low-latitudes. Figure 2 compares arbitrary longitudinal slices of cases $T 2, T 1, T 0$ and $Y_{2}^{2}$. Cases $T 0$ and $T 1$ satisfy (5) globally because the plumes driven from above extend radially and the azimuthal velocities peak below the Ekman layer at all latitudes. In case $T 2$ the velocities peak too deep at low-latitudes due to the strong mixing which breaks the radial plumes. Note that in this regime the dynamo is anyway non-dipolar (Kutzner and Christensen, 2002). In case $Y_{2}^{2}$ the perfect equatoriallysymmetric boundary condition favors rotational effects and the plumes extend along the $z$-direction rather than radially. However, the assumption holds well at all latitudes for the corresponding more relevant multi-harmonic boundary conditions case.

Another even more direct way of validating the relevance of the radial shear assumption (5) of the resulting Eqs. (6) and (7) consists in comparing the flows that those equations predict, with the numerical dynamo flows. This is done in Fig. 3 which shows two types of flows. The right column shows non-zonal time-average flows at the top of the core for four numerical dynamos with heterogeneous heat flux boundary conditions based on the lower mantle tomography model of Masters et al. (2000). The first flow model (the subcritical flow case $S$, Fig. 3(f)) is obtained from a stably-stratified rotating-convection simulation by assigning a Rayleigh number below the critical value for the onset of convection. This flow is in agreement with previous similar simulations (Gibbons and Gubbins, 2000). The next three flow models ( $T 0$, Fig. 3(g); $T 1$, Fig. 3(h); $T 2$, Fig. 3(i)) correspond to the full supercritical numerical dynamos we already discussed (from hereafter the dynamo steady flows). The dynamo steady flows should next be compared to their thermal wind counterparts computed by using Eqs. (6) and (7) assuming $L^{-1}=3$ (as suggested by Table 1), and relying on the exact time-average non-zonal density $\rho$ (as provided by the numerical dynamos). The left 
T2
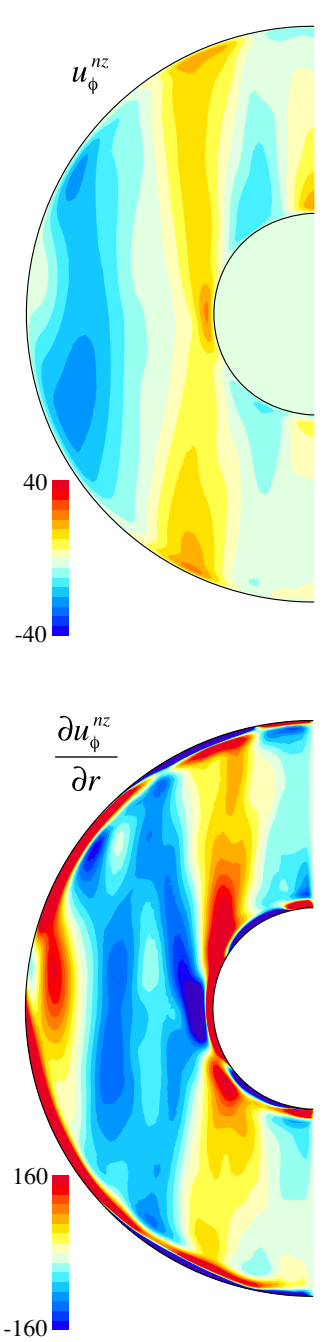

T1
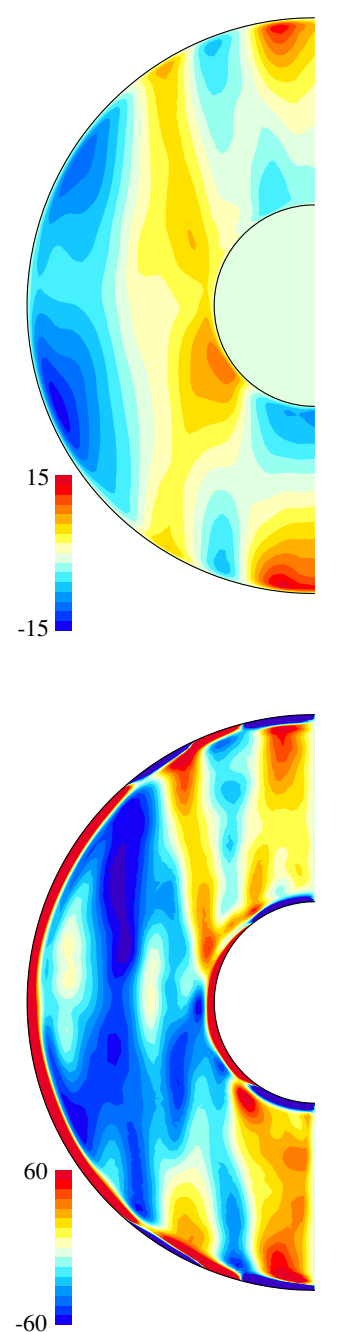

T0
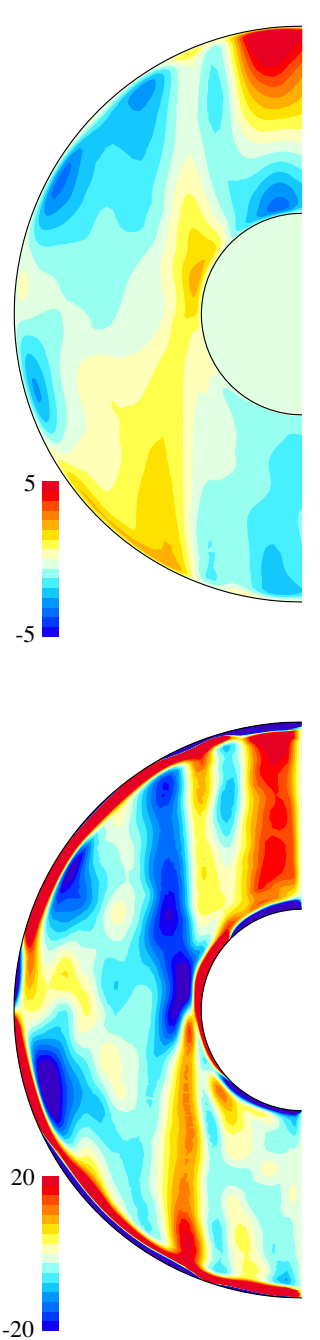

Y22
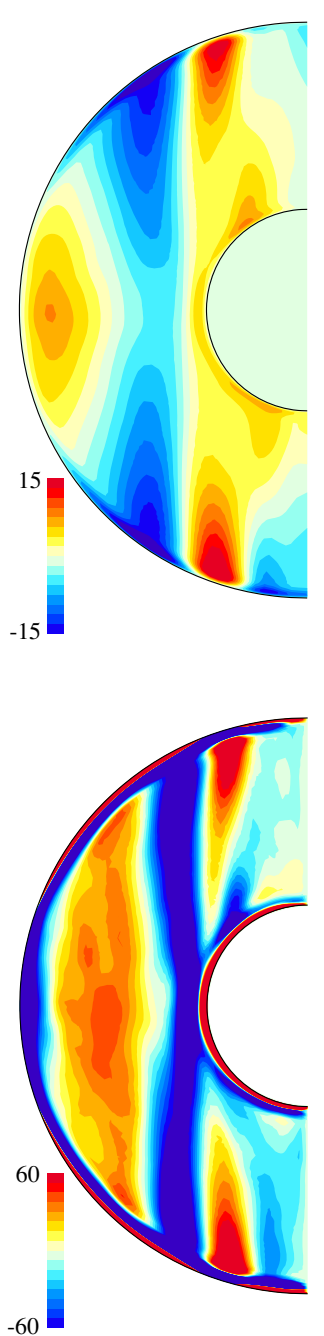

Fig. 2. Longitudinal slices of non-zonal time-average azimuthal flow $u_{\phi}^{\mathrm{nz}}$ (top) and non-zonal time-average radial shear of azimuthal flow ${\frac{\partial u_{\phi}}{\partial r}}^{\mathrm{nz}}$ (bottom) for cases $T 2, T 1, T 0$ and $Y_{2}^{2}$. Red/blue denotes eastward/westward flows or radial shears in dimensionless units. All slices are at the same longitude.

column of Fig. 3 shows those thermal wind flows with their driving density $\rho$. They very nicely show that if a correct knowledge of $\rho$ at the top of the free stream is available, relying on both the thermal wind Eqs. (3)-(4) and the radial shear assumption (5) to derive and use (6)-(7), allows to very closely predict the correct flow.

What about assumption (8)? This assumption also can be tested against numerical dynamos. Figure 3(j) shows the full lowermost mantle seismic shear velocity anomalies based on the tomographic model of Masters et al. (2000) which was used to impose the heat flux on the outer boundary for the numerical dynamos. Those led to the subcritical and dynamo steady flows shown in Figs. 3(f)-(i) and to the corresponding non-zonal density distributions shown in Figs. 3(a)-(d). Thus, checking that (8) is sensible essentially amounts to checking that the non-zonal tomographic pattern in Fig. 3(e) matches the non-zonal density patterns in Figs. 3(a)-(d). That is not exactly the case. Although all those density patterns have much in common (see $C_{\rho}$ values in Table 1), clear differences can be seen which translate into similar differences in the corresponding flow patterns (e.g. compare Figs. 3(c) and (h)). In particular, not all flow patterns display details such as the shift of about $15^{\circ}$ to the east in some southern hemisphere features with respect to their northern counterparts, which appear in the thermal wind model. Those are clearly related to the fact that (8) intrinsically implies some locking of the density pattern with respect to the heterogeneous heat flux boundary conditions we impose. Assumption (8) thus appears to be the weakest of the several assumptions our thermal wind model requires. This in fact, is not quite a surprise. Indeed, Olson and Christensen (2002) already found in numerical dynamos with a $Y_{2}^{2}$ boundary heat flux that the flow pattern tends to drift with respect to the boundary heterogeneity as a function of the Ekman number. However, Aubert et al. (2007) argued based on similar simulations that the drift is related to heat advection and is more likely a function of the Peclet number, with a maximum drift and distortion effects (in case of multi-harmonic boundary conditions) for intermediate values of this number. For very large Peclet numbers (relevant for Earth's core) this distortion is expected to remain small. For the purpose of the present study, we thus conclude that even though clearly not perfect, the thermal wind model proposed here is a reasonable reference model 
Thermal winds driven by dynamo densities with $\mathrm{L}^{-1}=3$
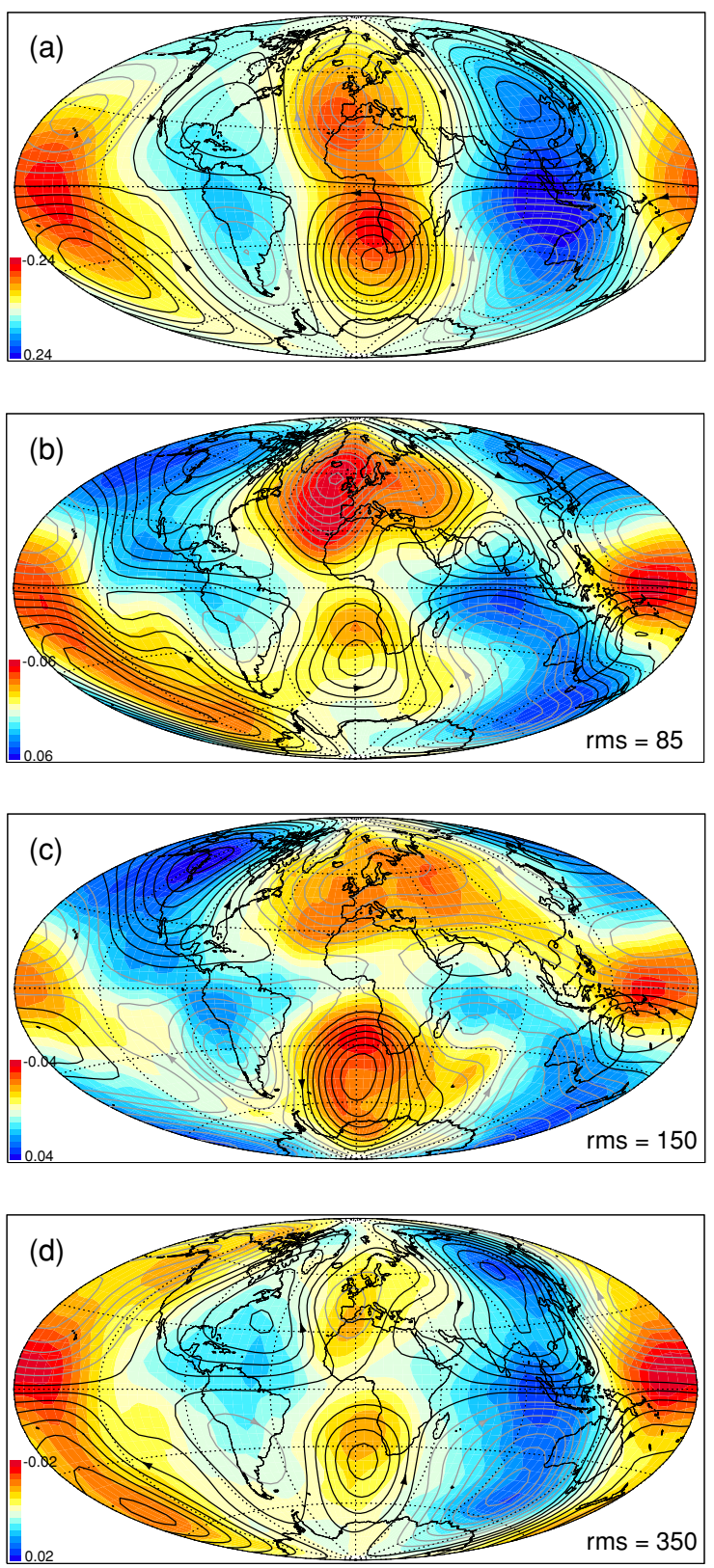

Tomographic thermal wind with $\mathrm{L}^{-1}=3$

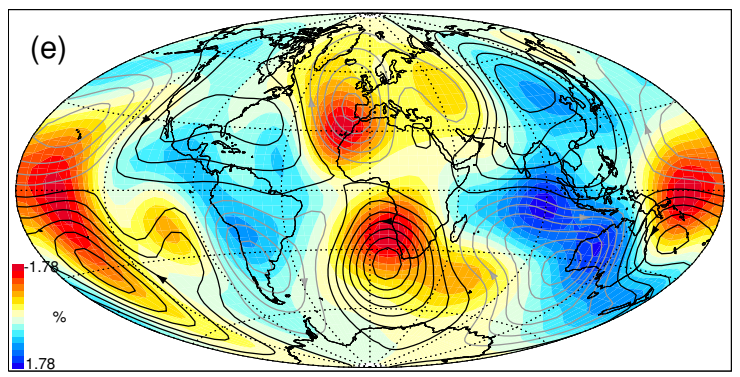

Dynamo flows
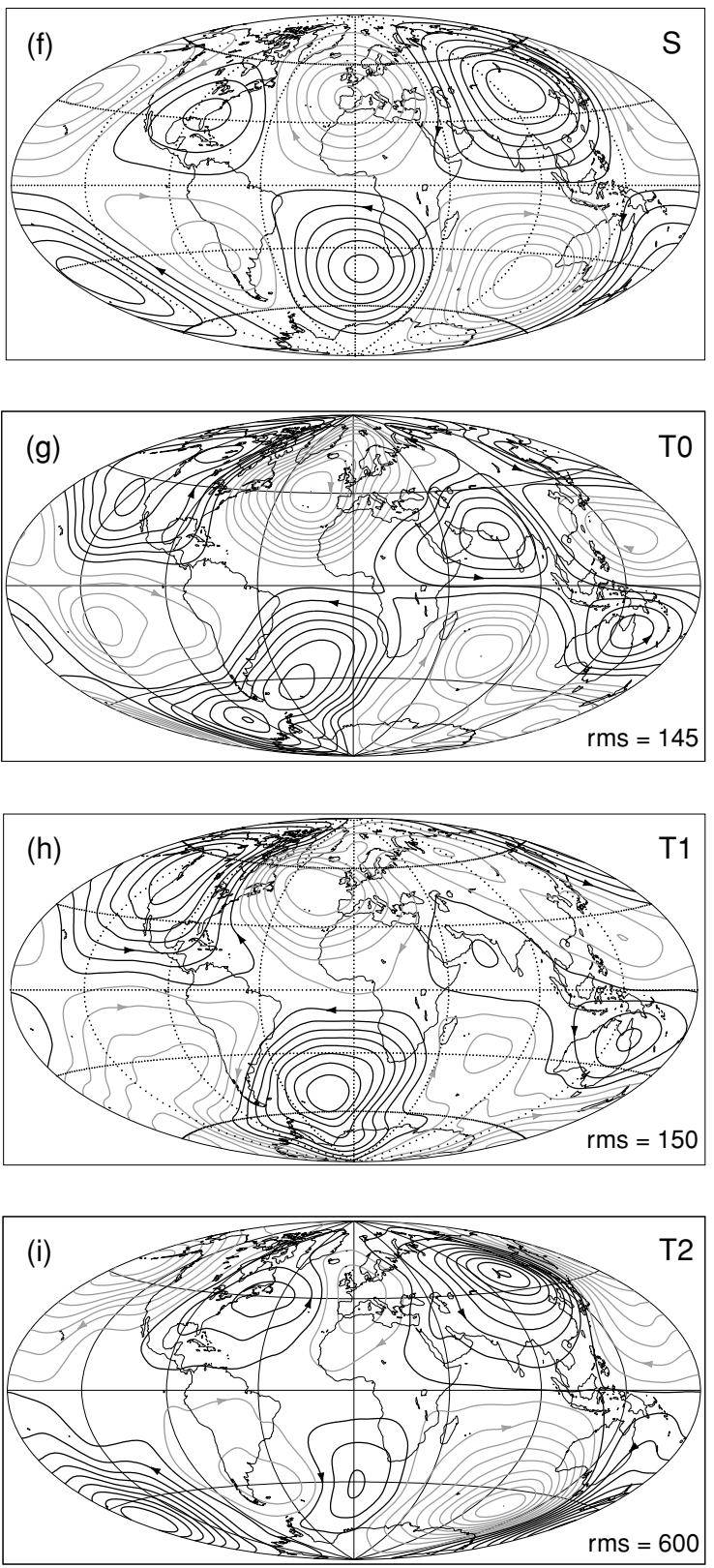

Full tomographic boundary conditions for the numerical dynamos

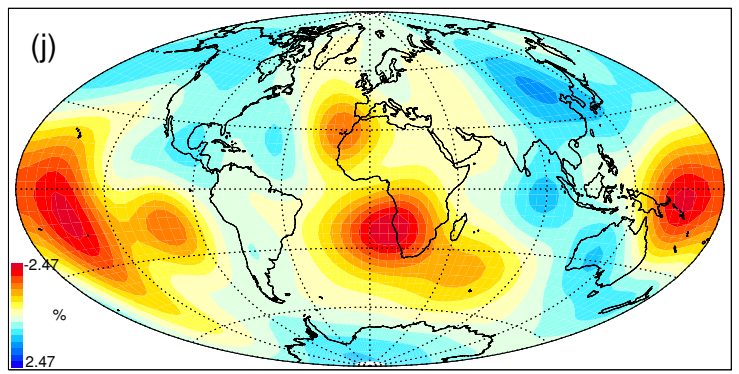

Fig. 3. Mantle-driven models of non-zonal core flow. (a)-(d): Non-zonal density at the top of the free stream from numerical dynamos, with superimposed streamfunction contours of the non-zonal thermal wind models for $L^{-1}=3$ and using those numerical dynamos non-zonal density. (f)-(i): The respective subcritical and dynamo steady flows. In (a)-(d) and (f)-(i): Contour intervals were adjusted for clear visualizations, flow rms of each column is given relative to case $S$ ((a) and (f) respectively). The cases from top to bottom are $S, T 0, T 1$ and $T 2$ (see Table 1 ). Black/grey contours denote anticlockwise/clockwise circulation, respectively. (e): Non-zonal seismic shear velocity model at lowermost mantle (Masters et al., 2000) truncated at spherical harmonic degree 8 , with superimposed streamfunction contours of the non-zonal thermal wind model for $L^{-1}=3$. Contour intervals were adjusted for clear visualizations. (j): Full seismic shear velocity model at lowermost mantle (Masters et al., 2000) truncated at spherical harmonic degree 8 used as a heat flux boundary condition for the numerical dynamos. In (e) and (j): Blue is positive $\delta v_{s} / v_{s}$ anomaly (dense, cold), red is negative (light, hot). 

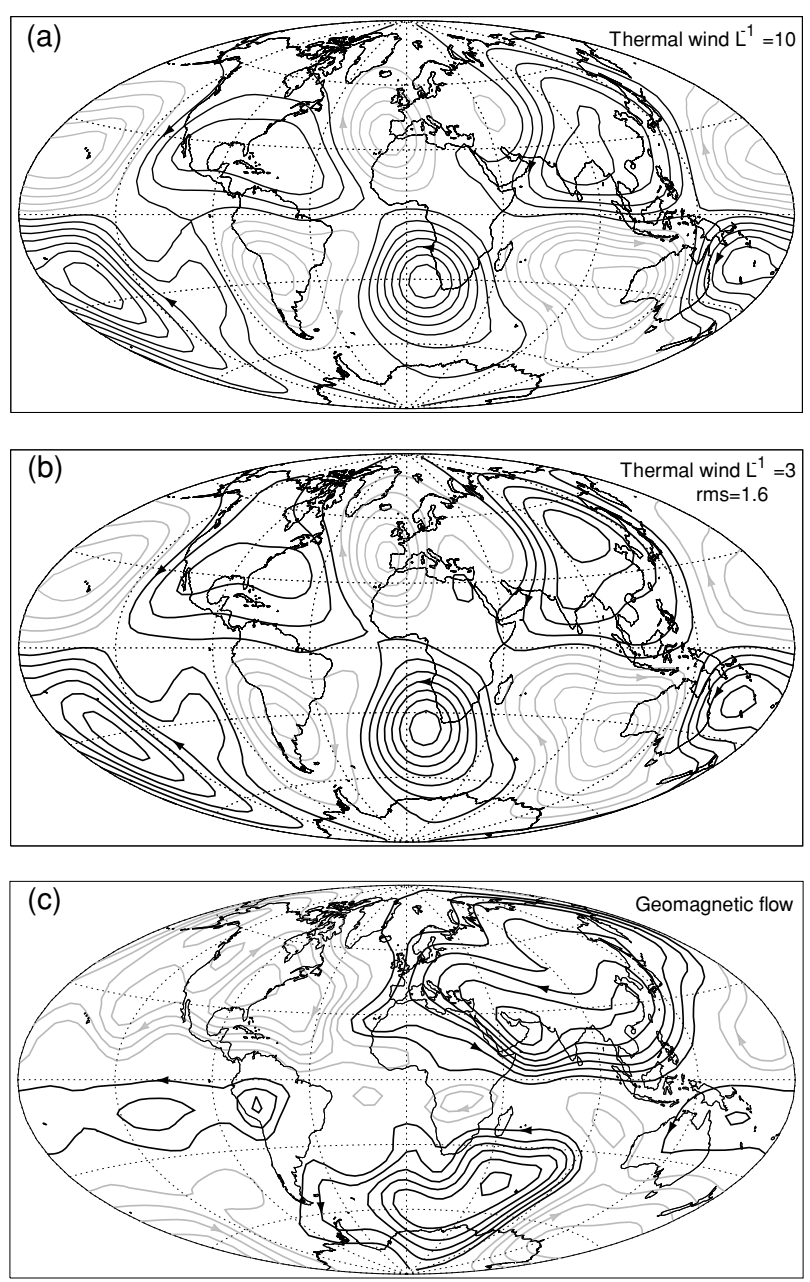

Fig. 4. Streamfunction of the non-zonal thermal wind model for $L^{-1}=10$ (a) and $L^{-1}=3(\mathrm{~b})$, and the non-zonal time-average geomagnetic flow (c), maximum velocity is $24.5 \mathrm{~km} /$ year, $\mathrm{rms}$ is $6.1 \mathrm{~km} /$ year). Flow rms in (b) is given relative to (a). In all maps black/grey contours denote anticlockwise/clockwise circulation, respectively. The vector correlation between (b) and (c) is 0.11 .

for investigating the influence of lower mantle thermal heterogeneity on the time-average non-zonal flow at the top of the core. We must however acknowledge that the differences seen among the various dynamo steady flows in Fig. 3 are likely reflections of the uncertainties one should expect for modelling mantle-driven flow with numerical dynamos, because of the limited computational ability to model the geodynamo process in the appropriate parameter regime.

It finally remains to point out that the choice of the value of the parameter $L^{-1}$ remains arbitrary. We chose $L^{-1}=3$ for the results shown in Fig. 3 motivated by our numerical dynamos (Table 1). But we also tried several other values. A larger value of $L^{-1}$ results in a weaker flow but does not much affect the pattern (see Figs. 4(a) and (b)). We conclude that the pattern of the thermal wind model is robust and does not depend greatly on our choice of the model parameter $L^{-1}$.

\section{Comparison with Time-average Flow Inferred from Geomagnetism}

We now compare the non-zonal thermal wind model with the non-zonal time-average core flow model of Amit and
Olson (2006) obtained from inversions of the geomagnetic secular variation model gufml (Jackson et al., 2000) over the period 1840-1990 (Fig. 4(c), from hereafter the geomagnetic flow). We chose this time interval since geomagnetic data prior to 1840 is considered less reliable because full magnetic vector measurements were not performed then. This flow model was obtained by solving the frozenflux radial magnetic induction equation using a helicalgeostrophic assumption (Amit and Olson, 2004, 2006) and averaging over the 150 years time period. The parameters used for this computation ( $k=0.15$ and $c=1)$ imply superimposed tangential geostrophy and helical flow constraints to ensure formal uniqueness of the solution. This, we note in Appendix B, is compatible with the thermal wind assumption. It leads to main flow features which are anyway also seen in other core flow models obtained from geomagnetic secular variation data using different approaches (Bloxham, 1989; Jackson et al., 1993; Holme, 1998; Chulliat and Hulot, 2000; Pais and Hulot, 2000; Hulot et al., 2002; Eymin and Hulot, 2005). Comparison between our thermal wind model (Fig. 4(b)) and the geomagnetic flow (Fig. 4(c)) reveals important agreements but also some discrepancies.

Main features in the non-zonal time-average geomagnetic flow include (1) a large anticlockwise vortex in midand high-latitudes of the southern hemisphere below the Indian and Atlantic Oceans, (2) a clockwise vortex below North America, (3) an anticlockwise vortex below Asia, and (4) a more active Atlantic hemisphere than the Pacific one. These features are quite persistent throughout the period considered, the rms of the non-zonal time-dependent part of the flow being only $30 \%$ of the rms of the non-zonal time-average flow.

Comparison with the thermal wind model shows that two of the main large-scale geomagnetic flow vortices are recovered by the thermal wind model in terms of both center and direction of circulation. The anticlockwise vortex below the Indian and Atlantic Oceans in the geomagnetic flow (Fig. 4(c)) is similar to the one found below the Southern Atlantic in the thermal wind model (Fig. 4(b)). The vortex below Asia associated with the high density mantle structure there is also in good agreement between the thermal wind model and the geomagnetic flow. In contrast, some flow features found in the thermal wind model, such as the vortex below North America, clearly disagree with the geomagnetic flow.

The thermal wind model predicts a large scale non-zonal core circulation that is nearly antisymmetric with respect to the equator, and is dominated by an azimuthal wavenumber $m=2$ pattern. However, a secondary $m=1$ component is also present. The centers of circulation in the southern hemisphere are shifted to the east by about $15^{\circ}$ longitude relative to their northern hemisphere counterparts. The subcritical and dynamo steady flows yield similar azimuthal wavenumber content and comparable shifts between the northern and southern hemispheres. In contrast, the geomagnetic flow shows a dominant $m=1$ with a secondary $m=2$ patterns, and a large eastward shift of the southern hemisphere structure relative to its northern hemisphere counterpart. The differences in azimuthal order content, in 

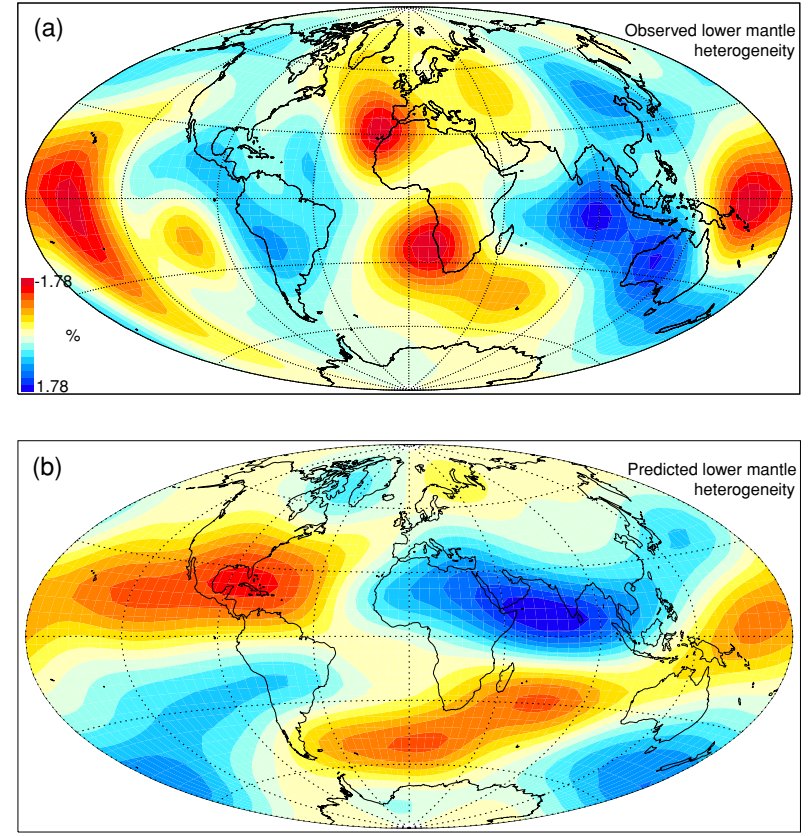

Fig. 5. Lower mantle density heterogeneity based on the observed seismic shear velocity tomographic model of Masters et al. (2000) (a), and the forward calculated density based on the geomagnetic flow and the thermal wind model (b). Blue is positive anomaly (dense, cold), red is negative (light, hot).

combination with the larger eastward shift in the southern hemisphere of the geomagnetic flow, result in good agreement between the thermal wind model and the geomagnetic flow over the eastern hemisphere of the core-mantle boundary, but relatively poor agreement over the western hemisphere.

Another interesting comparison can be made between the observed lower mantle seismic heterogeneity (which we assume drives the thermal wind) and the lower mantle seismic heterogeneity the geomagnetic flow would predict from Eqs. (6), (7) and (8) (Fig. 5). Regions of agreement include cold structures below the western limb of the Pacific rim and warm structures below the southern Atlantic, but discrepancies are also apparent, for example below North America where a seismic cold region is in disagreement with the forward calculated warm region. Overall, the spatial distribution of regions of agreement and discrepancy in heterogeneities (Fig. 5) is similar to the comparison of the two flow models (Fig. 4), with more agreement in the eastern hemisphere than in the western hemisphere.

What could be the reasons for such discrepancies? First, thermal wind could of course be an oversimplified theory for steady core flow. Although our analysis of numerical dynamos suggests that the thermal wind balance is wellsatisfied, whether this also holds for the Earth's core can still be questioned. Second, the input we use for the thermal core-mantle boundary conditions is clearly simplified and idealized. Part of the buoyancy in the lower mantle may be attributed to chemical (Trampert et al., 2004) or mineralogical (Murakami et al., 2004) anomalies. Third, various problems associated with core flow inversions from geomagnetic data (Bloxham and Jackson, 1991; Eymin and Hulot, 2005; Holme, 2007), especially nonuniqueness is- sues, might result in inaccurate core flow models for comparison with thermal wind models. In this respect, and as pointed out to us by R. Holme (personnal communication), the extent to which the steady part of a flow model inferred from geomagnetic data could be found with a driving density forced to be significantly close to that inferred from lower mantle seismology, would clearly be worth investigating further. Finally, the time interval used for averaging the geomagnetic flow, 150 years, might still be too short.

All of the above discussion was based on comparisons of flow patterns and ignored the value of the parameter $C$ in (8). We may now infer an estimate of $C$ based on our thermal wind model. By constraining rms magnitudes for the thermal wind model and the geomagnetic flow to be equal, and using $\Omega=7.29 \cdot 10^{-5} \mathrm{~s}^{-1}$ and $g_{0}=10.68 \mathrm{~m} \mathrm{~s}^{-2}$ (Dziewonsky and Anderson, 1981), we find $C \sim 2.7 \cdot 10^{-7}$. This value is consistent with $\delta v_{s} / v_{s} \sim 2 \cdot 10^{-2}$ (Masters et al., 2000) while the Boussinesq approximation on the core side requires $\delta \rho / \rho_{0}=\alpha \delta T$ with thermal expansivity $\alpha \sim 10^{-5} \mathrm{~K} \mathrm{~s}^{-1}$ (Poirier, 2000) and temperature anomaly $\delta T \sim 10^{-3} \mathrm{~K}$ (Bloxham and Gubbins, 1987).

In summary, we have introduced a simple thermal wind model to predict the steady flow at the top of the core from heterogeneous boundary conditions imposed by the lower mantle. This thermal wind model has been validated using numerical dynamos. It provides an easy way to investigate the likely signature of thermal boundary conditions imposed by the mantle. Using the model of Masters et al. (2000) to infer thermal mantle heterogeneity, the thermal wind model shares several important features with a time-average flow inferred from 150 years of geomagnetic data (Amit and Olson, 2006). Discrepancies might originate from incomplete interpretation of the seismic data, uncertainties in core flow modelling, or insufficient timeaveraging of the geomagnetic core flow. Future progress in modelling mantle tomography, geomagnetic secular variation and core flow may provide further insight to the understanding of core-mantle interactions and the steady flow in the core.

Acknowledgments. Numerical calculations were performed at the Service de Calcul Parallelle, IPGP. H. A. was supported by a grant from the IntraEuropean MarieCurie Action. We thank Richard Holme and Alexandra Pais for their useful reviews. This is IPGP contribution 2384.

\section{Appendix A. Method}

We calculate the right hand side of (6)-(7) using the mantle density model and the thermal core-mantle coupling assumption (8). We solve for each flow component using a matrix inversion method. At the closest latitudes to the poles we use the smallness of $\sin \theta$ to neglect the meridional derivative terms, and by that we avoid a problem of undefined polar velocities in spherical coordinates. To avoid numerical instabilities at the equator, we solve for each hemisphere separately by setting a matching boundary condition at the equator. For the azimuthal velocity, we use the exact form of (7) at the equator,

$$
-\left.\frac{\partial u_{\theta}}{\partial \theta}\right|_{\mathrm{eq}}=\left.\frac{g_{0}}{2 \Omega \rho_{0}} \frac{\partial \rho}{\partial \phi}\right|_{\mathrm{eq}}
$$


Our goal is to find the (main) toroidal flow, so we assume non-divergence at the equator,

$$
\left.\frac{\partial u_{\theta}}{\partial \theta}\right|_{\mathrm{eq}}=-\left.\frac{\partial u_{\phi}}{\partial \phi}\right|_{\mathrm{eq}}
$$

Combining (A.1) and (A.2) we get the approximated boundary condition for the azimuthal flow:

$$
\left.u_{\phi}\right|_{\mathrm{eq}}=\left.\frac{g_{0}}{2 \Omega \rho_{0}} \rho\right|_{\mathrm{eq}}
$$

For the meridional velocity, we set $\left.u_{\theta}\right|_{\text {eq }}=0$ at the equator, which is the exact solution to the radial component of the thermal wind, generally known as the tangential geostrophic constraint.

Introducing $\theta_{i}=\theta_{0}+i \delta$ from $\theta_{0}=2.5^{\circ}$ to $\theta_{n}=$ $87.5^{\circ}$ with $\delta=5^{\circ}$, the thermal wind equation for each flow component $\left(u_{\phi}\right.$ and $\left.u_{\theta}\right)$ at longitude $\phi$ in the northern hemisphere can then be discretized and written in the matrix form:

$$
\mathbf{M} u=b
$$

where $u$ is either $\left(u_{\theta}\left(\theta_{0}\right), u_{\theta}\left(\theta_{1}\right), \ldots u_{\theta}\left(\theta_{n}\right)\right)$ when considering the meridional flow component $u_{\theta}$, or $\left(u_{\phi}\left(\theta_{0}\right), u_{\phi}\left(\theta_{1}\right)\right.$, $\ldots u_{\phi}\left(\theta_{n}\right)$ ) when considering the azimuthal flow component $u_{\phi}$, and $\mathbf{M}$ is given by (accounting for the boundary conditions):

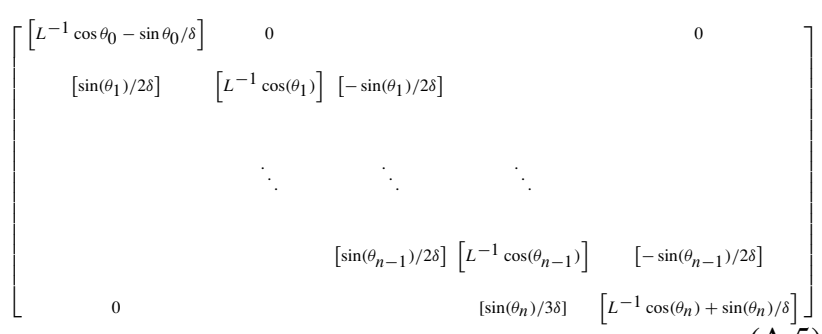

The vector $b$ is then given either by

$$
\frac{g_{0}}{2 \Omega \rho_{o}}\left[\begin{array}{c}
-\partial \rho / \partial \theta\left(\theta_{0}\right) \\
-\partial \rho / \partial \theta\left(\theta_{1}\right) \\
\vdots \\
-\partial \rho / \partial \theta\left(\theta_{n-1}\right) \\
-\partial \rho / \partial \theta\left(\theta_{n}\right)+(4 / 3 \delta) \sin \left(\theta_{n}\right) \rho_{\mathrm{eq}}
\end{array}\right]
$$

when considering $u \equiv u_{\phi}$, or

$$
\frac{g_{0}}{2 \Omega \rho_{o}}\left[\begin{array}{c}
\left(1 / \sin \left(\theta_{0}\right)\right) \partial \rho / \partial \phi\left(\theta_{0}\right) \\
\left(1 / \sin \left(\theta_{1}\right)\right) \partial \rho / \partial \phi\left(\theta_{1}\right) \\
\vdots \\
\left(1 / \sin \left(\theta_{n-1}\right)\right) \partial \rho / \partial \phi\left(\theta_{n-1}\right) \\
\left(1 / \sin \left(\theta_{n}\right)\right) \partial \rho / \partial \phi\left(\theta_{n}\right)
\end{array}\right]
$$

when considering $u \equiv u_{\theta}$, and $\rho_{\mathrm{eq}}$ is the density at the equator. For each longitude $\phi$, and each flow component $u_{\phi}$ and $u_{\theta}$, the matrix $\mathbf{M}$ is tri-diagonal and therefore invertible. Solutions are then obtained through

$$
u=\mathbf{M}^{-1} b
$$

A similar procedure is used to compute the flow in the southern hemisphere.

\section{Appendix B. Compatibility of the Helical- geostrophic and Thermal Wind Assumptions}

The thermal wind equation is the $3 \mathrm{D}$ balance of Coriolis and buoyancy vorticities (1). Its tangential components (3) and (4) retain both contributors, but the radial buoyancy vorticity is identically zero, yielding the tangential geostrophic equation: $\nabla_{h} \cdot\left(\vec{u}_{h} \cos \theta\right)=0$. This suggests that the thermal wind assumption should be more compatible with core flows derived with the tangential geostrophic assumption than with an helical-geostrophic assumption. However, numerical dynamos prove that the tangential components (3) and (4) of the thermal wind balance (1) (Aubert, 2005; Aubert et al., 2007) and the helical flow assumption (Olson et al., 2002; Amit et al., 2007) may both be reasonably satisfied. In numerical dynamos, helicity is generated by a temperature field configuration that produces thermal winds with peak $z$-velocities close to the center of columnar vortices (Olson et al., 1999). On approach to the outer surface, the $z$-velocities turn to tangentially divergent flow, which correlates with radial vorticity at the top of the free stream. The radial Coriolis vorticity is expected to be smaller than its tangential counterparts, and may be relaxed by radial viscous vorticity. This relaxation may lead to the shift between upwellings at centers of vortices (according to helical flow) and upwellings at the east/west limbs of vortices (according to tangential geostrophy). It is therefore reasonable to compare the thermal wind flow models with core flows inverted based on the helical flow assumption.

\section{References}

Amit, H. and P. Olson, Helical core flow from geomagnetic secular variation, Phys. Earth Planet. Inter., 147, 1-25, 2004.

Amit, H. and P. Olson, Time-average and time-dependent parts of core flow, Phys. Earth Planet. Inter., 155, 120-139, 2006.

Amit, H., P. Olson, and U. Christensen, Tests of core flow imaging methods with numerical dynamos, Geophys. J. Int., 168, 27-39, 2007.

Aubert, J., Steady zonal flows in spherical shell fluid dynamos, J. Fluid Mech., 542, 53-67, 2005.

Aubert, J., H. Amit, and G. Hulot, Detecting thermal boundary control in surface flows from numerical dynamos, Phys. Earth Planet. Inter., 160, 143-156, 2007.

Bloxham, J., Simple models of fluid flow at the core surface derived from geomagnetic field models, Geophys. J. Int., 99, 173-182, 1989.

Bloxham, J. and D. Gubbins, Thermal core-mantle interactions, Nature, 325, 511-513, 1987.

Bloxham, J. and A. Jackson, Fluid flow near the surface of the Earth's outer core, Rev. Geophys., 29, 97-120, 1991.

Bouligand, C., G. Hulot, A. Khokhlov, and G. Glatzmaier, Statistical paleomagnetic field modeling and dynamo numerical simulation, Geophys. J. Int., 161, 603-626, 2005.

Carlut, J. and V. Courtillot, How complex is the time-averaged geomagnetic field over the past 5 Myr?, Geophys. J. Int., 134, 527-544, 1998.

Christensen, U. and P. Olson, Secular variation in numerical geodynamo models with lateral variations of boundary heat flow, Phys. Earth Planet. Inter., 138, 39-54, 2003.

Chulliat, A. and G. Hulot, Local computation of the geostrophic pressure at the top of the core, Phys. Earth Planet. Inter., 117, 309-328, 2000.

Constable, C., C. Johnson, and S. Lund, Global geomagnetic field models for the past 3000 years: transient or permanent flux lobes?, Phil. Trans. Roy. Soc. A, 358, 991-1008, 2000.

Dziewonsky, A. M. and D. L. Anderson, Preliminary reference Earth model, Phys. Earth Planet. Inter., 25, 297-356, 1981.

Eymin, C. and G. Hulot, On surface core flows inferred from satellite magnetic data, Phys. Earth Planet. Inter., 152, 200-220, 2005.

Forte, A. M. and J. X. Mitrovica, Deep-mantle high-viscosity flow and thermochemical structure inferred from seismic and geodynamic data, 
Nature, 410, 1049-1056, 2001

Gibbons, S. J. and D. Gubbins, Convection in the earths core driven by lateral variations in the core-mantle boundary heat flux, Geophys. J. Int., 142, 631-642, 2000 .

Glatzmaier, G., R. Coe, L. Hongre, and P. Roberts, The role of the earth's mantle in controlling the frequency of geomagnetic reversals, Nature, 401, 885-890, 1999.

Gubbins, D., Thermal core-mantle interactions: theory and observations, in Earth's Core: dynamics, structure and rotation, edited by V. Dehant, K. Creager, S. Karato, and S. Zatman, 277 pp., AGU Geodynamics Series American Geophysical Union, Washington D.C., 2003.

Gubbins, D. and P. Kelly, Persistent patterns in the geomagnetic field over the past 2.5 Myr, Nature, 365, 829-832, 1993.

Holme, R., Electromagnetic core-mantle coupling-I. Explaining decadal changes in the length of day, Geophys. J. Int., 132, 167-180, 1998.

Holme, R., Large-scale Flow in the Core, in Treatise on Geophysics vol. 8 , edited by Olson, P., 358 pp., Elsevier Science, London, 2007.

Hongre, L., G. Hulot, and A. Khokhlov, An analysis of the geomagnetic field over the past 2000 years, Phys. Earth Planet. Inter., 106, 311-335, 1998.

Hulot, G. and J.-L. LeMouël, A statistical approach to the Earth's main magnetic field, Phys. Earth Planet. Inter., 82, 167-183, 1994.

Hulot, G. and C. Bouligand, Statistical paleomagnetic field modeling and symmetry considerations, Geophys. J. Int., 161, 591-602, 2005.

Hulot, G., C. Eymin, B. Langlais, M. Mandea, and N. Olsen, Small-scale structure of the geodynamo inferred from Oersted and Magsat satellite data, Nature, 416, 620-623, 2002.

Jackson, A., J. Bloxham, and D. Gubbins, in Dynamics of Earth's deep interior and Earth rotation, edited by LeMouël, J.-L., D. E. Smylie, and T. Herring, 189 pp., Geophysical Monograph 72 IUGG, Washington D.C., 1993.

Jackson, A., A. R. T. Jonkers, and M. R. Walker, Four centuries of geomagnetic secular variation from historical records, Phil. Trans. R. Soc. Lond., A358, 957-990, 2000.

Johnson, C. and C. Constable, The time averaged geomagnetic field as recorded by lava flows over the past $5 \mathrm{Myr}$, Geophys. J. Int., 112, 489$519,1995$.

Khokhlov, A., G. Hulot, and C. Bouligand, Testing statistical paleomagnetic field models against directional data affected by measurement errors, Geophys. J. Int., 167, 635-648, 2006.

Korte, M., A. Genevey, C. Constable, U. Frank, and E. Schnepp, Continuous geomagnetic field models for the past 7 millenia: 1 . A new global data compilation, Geochem. Geophys. Geosyst., 6 , doi:10.1029/2004GC000800, 2005.
Kutzner, C. and U. Christensen, From stable dipolar towards reversing numerical dynamos, Phys. Earth Planet. Inter., 131, 29-45, 2002.

Le Huy, M., J.-L. LeMouël, and A. Pais, Time evolution of the fluid flow at the top of the core. Geomagnetic jerks, Earth Planets Space, 52, 163173, 2000.

Masters, G., G. Laske, H. Bolton, and A. Dziewonski, in Earth's deep interior, edited by S. Karato, A. M. Forte, R. C. Liebermann, G. Masters, and L. Stixrude, 289 pp., AGU monograph 117, Washington D.C., 2000.

McElhinny, M., P. McFadden, and R. Merrill, The time-averaged paleomagnetic field 0-5 ma, J. Geophys. Res., 101, 25,007-25,027, 1996.

Murakami, M., K. Hirose, K. Kawamura, N. Sata, and Y. Ohishi, PostPerovskite Phase Transition in $\mathrm{MgSiO}_{3}$, Science, 304, 855-858, 2004.

Olson, P. and U. Christensen, The time averaged magnetic field in numerical dynamos with nonuniform boundary heat flow, Geophys. J. Int., 151, 809-823, 2002.

Olson, P., U. R. Christensen, and G. A. Glatzmaier, Numerical modeling of the geodynamo: mechanisms of field generation and equilibration, $J$. Geophys. Res., 104, 10383-10404, 1999.

Olson, P. and G. A. Glatzmaier, Magnetoconvection and thermal coupling of the Earth's core and mantle, Phil. Trans. R. Soc. Lond., A354, 14131424, 1996.

Olson, P., I. Sumita, and J. Aurnou, Diffusive magnetic images of upwelling patterns in the core, J. Geophys. Res., 107, 2348, 2002.

Pais, A. and G. Hulot, Length of day decade variations, torsional oscillations and inner core superrotation: evidence from recovered core surface zonal flows, Phys. Earth Planet. Inter., 118, 291-316, 2000.

Pedlosky, J., Geophysical Fluid Dynamics, 710 pp., Springer, New York, 1987.

Poirier, J.-P., Introduction to the Physics of the Earth's Interior, 312 pp., Cambridge University Press, Cambridge, UK, 2000.

Rau, S., U. Christensen, A. Jackson, and J. Wicht, Core flow inversion tested with numerical dynamo models, Geophys. J. Int., 141, 485-497, 2000 .

Trampert, J., F. Deschamps, J. Resovsky, and D. Yuen, Probabilistic Tomographic Maps Chemical Heterogeneities Throughout the Lower Mantle, Science, 306, 853-856, 2004.

Yuen, D. A., O. Cadek, A. Chopelas, and C. Mtyska, Geophysical inferences of thermal-chemical structures in the lower mantle, Geophys. Res. Lett., 20, 899-902, 1993.

H. Amit (e-mail: hagay@ipgp.jussieu.fr), J. Aubert, G. Hulot, and P. Olson 